\title{
Traditional and emerging technologies for washing and volume reducing blood products
}

This article was published in the following Dove Medical Press journal: Journal of Blood Medicine

\author{
Madeleine Lu \\ Dalia L Lezzar \\ Eszter Vörös \\ Sergey S Shevkoplyas \\ Department of Biomedical \\ Engineering, University of Houston, \\ Houston, TX 77204-5060, USA
}

Correspondence: Sergey S Shevkoplyas Department of Biomedical Engineering, University of Houston, 3605 Cullen Blvd, Houston, TX 77204-5060, USA

$\mathrm{Tel}+\mathrm{I} 7 \mathrm{I} 37435696$

$\mathrm{Fax}+\mathrm{I} 7137430226$

Email sshevkoplyas@uh.edu

\begin{abstract}
Millions of blood components including red blood cells, platelets, and granulocytes are transfused each year in the United States. The transfusion of these blood products may be associated with adverse clinical outcomes in some patients due to residual proteins and other contaminants that accumulate in blood units during processing and storage. Blood products are, therefore, often washed in normal saline or other media to remove the contaminants and improve the quality of blood cells before transfusion. While there are numerous methods for washing and volume reducing blood components, a vast majority utilize centrifugation-based processing, such as manual centrifugation, open and closed cell processing systems, and cell salvage/autotransfusion devices. Although these technologies are widely employed with a relatively low risk to the average patient, there is evidence that centrifugation-based processing may be inadequate when transfusing to immunocompromised patients, neonatal and infant patients, or patients susceptible to transfusion-related allergic reactions. Cell separation and volume reduction techniques that employ centrifugation have been shown to damage blood cells, contributing to these adverse outcomes. The limitations and disadvantages of centrifugation-based processing have spurred the development of novel centrifugation-free methods for washing and volume reducing blood components, thereby causing significantly less damage to the cells. Some of these emerging technologies are already transforming niche applications, poised to enter mainstream blood cell processing in the not too distant future.
\end{abstract}

Keywords: washing, volume reduction, red blood cells, platelets, granulocytes, transfusion

\section{Introduction}

Millions of blood components including red blood cells (RBCs), platelets, and granulocytes are transfused each year in the United States. The transfusion of stored blood products has been associated with adverse clinical outcomes ${ }^{1-3}$ brought on by residual plasma proteins, excess potassium, free hemoglobin, and other contaminants that accumulate during storage. ${ }^{4-6}$ To avoid these effects, washing or volume reduction of blood products is often performed to remove contaminants and improve the quality of a unit before transfusion. These procedures conventionally use centrifugation-based cell processors that separate blood cells from the suspending medium based on the difference in their respective densities. Although widely used, these cell processors have limitations that may outweigh their advantages in some settings. There has, therefore, been a significant research effort in recent years for the development of novel centrifugation-free approaches to wash and volume reduce blood products.

Volume reduction is conventionally performed through the centrifugation of a blood component and the subsequent removal of the supernatant. A reduction in sample 
volume is often required in instances of platelet transfusions, specifically for neonates and infants, where the recipient is unable to withstand transfusions of large volumes. ${ }^{7}$ Volume reduction is also used for patients prone to severe transfusionrelated allergic reactions brought on by plasma proteins and inflammatory mediators released by residual white blood cells (WBCs). ${ }^{8,9}$ Blood washing involves the dilution of a blood component and subsequent volume reduction of the diluted unit to a desired concentration of blood cells. While stored RBCs are the most common washed blood product, platelet washing is also indicated for neonates receiving transfusions, patients undergoing chemotherapy, and patients prone to transfusion-related allergic reactions. ${ }^{1,2}$ Whole blood is washed during cell salvage procedures to remove foreign bodies and contaminants suctioned from the incision site. Any blood product cryopreserved to prolong its shelf life is also washed to remove toxic preservatives. ${ }^{10,11}$ The ultimate goal of volume reduction and washing is to provide an adequately pure sample of a component of blood for transfusion or transplantation, with reduced risk for adverse clinical outcomes. Here we present various methods for blood washing and volume reduction of cellular blood products and highlight new developments in this field (advantages and disadvantages of the technologies are summarized in Table 1).

\section{Centrifugation-based technologies Manual centrifugation}

Manual volume reduction and washing typically involves pelleting blood cells in a unit using a swinging bucket centrifuge, and discarding the supernatant. As previously mentioned, neonates and infants require volume-reduced platelet concentrates to avoid large transfusion volumes and reduce the risk of fetomaternal alloimmune thrombocytopenia. Volume reduction can also reduce transfusion-related allergic reactions in susceptible patients. ${ }^{12}$ Since there is currently no standard for the preparation of volume reduced platelets, manual centrifugation using a high-capacity centrifuge is most often used. ${ }^{13,14}$ However, since volume reduction procedures are not standardized, there are varying spin speeds reported to concentrate platelets. The mechanical stresses placed on platelets, via centrifugal forces, have been shown to lead to significant platelet activation. ${ }^{15}$ Therefore, discrepancies in spin speeds make the process of manual centrifugation less robust and produces platelet concentrates with wide ranges of quality and purity. ${ }^{16}$ Furthermore, the manual centrifugation of blood components adds an additional layer of user manipulation, increasing the risk of contamination. ${ }^{12}$ While the procedure of manually washing platelets is somewhat laborious and has limitations, when performed correctly, the process can yield high recoveries at a fraction of the cost of automated processing systems. ${ }^{15}$

\section{Automated systems: open and closed}

Most blood banking centers utilize automated systems to wash or volume reduce the blood products they produce and distribute. Automated systems are pre-programmed with a variety of cell processing procedures, from density gradient separation to the deglycerolizing of thawed products. In a survey of 15 children's hospitals (150-400 beds) across America, 14 out of 15 hospitals used the COBE 2991 cell processor (Terumo BCT, Lakewood, CO, USA), making it one of the most popular processors in the field. ${ }^{17}$ This centrifugation system separates each blood component by their respective density and each layer of fluid is expressed from the disposable insert by connected tubing. After separation, normal saline can be added to dilute the blood product, which is then removed via a connector tubing to obtain a volumereduced and/or washed product.

RBCs are known to experience significant deterioration while in storage leading to leakage of potassium and free hemoglobin into the supernatant. ${ }^{6,18}$ For this reason, transfusion of older units (stored longer than 7 days) is often not recommended for neonates and infants undergoing cardiac surgery, in order to prevent hyperkalemic cardiac arrest and other complications. Transfusion of older units is also contraindicated for infants born preterm who suffer from severe anemia. ${ }^{19,20}$ Washing RBC units using COBE 2991 has been shown to reduce pro-inflammatory markers and to decrease the number of transfusions necessary for pediatric patients undergoing cardiac bypass. ${ }^{21}$ Cortés-Puch et al also found, in a canine study, that washing RBC units approaching their expiration date (42 days) using the COBE 2991 system improves outcomes when compared to unwashed 42-day-old RBC units. ${ }^{22}$ This result indicates that washing older units for later transfusion can be advantageous when the supply and availability of fresh blood is low. This same study, however, also found that washing 7-day-old RBCs led to increased organ failure and mortality and that the quality of 42-day-old RBCs could not be restored to the level of the 7-day-old, unwashed RBCs. ${ }^{22}$ These findings suggest that the COBE 2991 washing system should be reserved for RBC units nearing the end of the allowable storage.

Although stored RBCs washed using the COBE 2991 can be transfused within 24 hours, O'Leary et al found that the concentration of extracellular potassium in washed units at 24 hours actually exceeds prewash potassium levels, and 
Table I A summary of the advantages and disadvantages of each of the technologies described in this study

\begin{tabular}{|c|c|c|c|c|}
\hline \multicolumn{3}{|l|}{ Technology } & Pros & Cons \\
\hline \multicolumn{3}{|l|}{ Manual } & Low $\operatorname{cost}^{14,15}$ & $\begin{array}{l}\text { Higher risk of contamination; }{ }^{12} \text { no } \\
\text { standardized procedures; } ;{ }^{1,14,16} \text { laborious }\end{array}$ \\
\hline \multirow[t]{2}{*}{ Automated } & \multicolumn{2}{|l|}{ COBE 299I } & $\begin{array}{l}\text { Most commonly used; }{ }^{17} \text { effectively reduces } \\
\text { pro-inflammatory markers; }{ }^{21} \text { restores } \\
\text { quality of RBC units nearing expiration }{ }^{22}\end{array}$ & $\begin{array}{l}\text { Significant increase in potassium and } \\
\text { hemolysis found post-wash:; ;,23 ineffectively } \\
\text { reduces DMSO on cryopreserved } \\
\text { products }^{24}\end{array}$ \\
\hline & \multicolumn{2}{|l|}{ ACP 215} & $\begin{array}{l}\text { Reduces risk of bacterial contamination; }{ }^{25} \\
\text { allows the addition of additive solution to } \\
\text { extend shelf life }{ }^{25,26}\end{array}$ & $\begin{array}{l}\text { Unable to consistently reduce lgA levels } \\
\text { for IgA-deficient patients; }{ }^{30,31} \text { activates } \\
\text { platelets and } \mathrm{WBCs}^{34,35}\end{array}$ \\
\hline \multirow[t]{2}{*}{ Autotransfusion } & Discontinuous & $\begin{array}{l}\text { Cell saver Elite, } \\
\text { Cell Saver } 5 \text {, } \\
\text { and OrthoPAT }\end{array}$ & $\begin{array}{l}\text { On par with conventional cell processors } \\
\text { for removing potassium, free hemoglobin, } \\
\text { platelet, and WBC from whole blood; }{ }^{37} \text { can } \\
\text { process variety of volumes }{ }^{43}\end{array}$ & $\begin{array}{l}\text { Activates cells that adhere to the bowl } \\
\text { and releases leukoattractants and } \\
\text { thromboplastic materials; } ;^{27} \text { increases } \\
\text { hemolysis and microparticles in } \\
\text { solution; }{ }^{40,50} \text { has significant dead volume; }{ }^{43,44} \\
\text { has varying process times; }{ }^{43,44} \text { exhibits } \\
\text { low platelet recovery from platelet-rich } \\
\text { plasma }^{51,52}\end{array}$ \\
\hline & Continuous & CATS & $\begin{array}{l}\text { Produces RBC concentrates of a high } \\
\text { hematocrit; }{ }^{37,45} \text { all processing occurs in one } \\
\text { chamber; can process small volumes; has } \\
\text { limited dead volume; effectively removes } \\
\text { contaminants (eg, fat and mircoaggregates) }\end{array}$ & $\begin{array}{l}\text { Provides inconsistent hemoglobin } \\
\text { removal; } ; 1 \text { has negative effect on RBC } \\
\text { deformability and cell } 2,3-\mathrm{DPG}^{47}\end{array}$ \\
\hline \multirow[t]{3}{*}{ Membrane filters } & Hollow fiber & $\begin{array}{l}\text { PSN I20, } \\
\# 23.05, \\
\text { Sangofer, } \\
\text { ErySep, and 3M }\end{array}$ & $\begin{array}{l}\text { Effectively removes DMSO (PSN I20),53 } \\
\text { separates RBC from whole placental blood } \\
\text { when attached to a leukoreduction filter } \\
\text { (Sangofer, }{ }^{54} 3 M^{55} \text { and ErySep }{ }^{56} \text { ) }\end{array}$ & $\begin{array}{l}\text { Causes platelet activation, }{ }^{53} \text { exhibits low } \\
\text { platelet recovery; } ; 5-58 \text { significantly reduces } \\
\text { clotting factors; }{ }^{55-58} \text { unable to significantly } \\
\text { remove DMSO }(\# 23.05)^{53}\end{array}$ \\
\hline & Physical & $\begin{array}{l}\text { PAF and } \\
\text { HemoSep }\end{array}$ & $\begin{array}{l}\text { Reduces amount of target solute (eg, } \\
\text { potassium }{ }^{59} \text { and ammonium); ;1 low cost } \\
\text { and portable for resource-limited settings } \\
(\text { HemoSep })^{67,68}\end{array}$ & $\begin{array}{l}\text { Filters not compatible with current rapid } \\
\text { blood transfusion devices; }{ }^{62} \text { little research } \\
\text { on RBC damage from PAF performed; } 22,63 \\
\text { subpar RBC concentration from HemoSep } \\
\text { in comparison to CATS }{ }^{69}\end{array}$ \\
\hline & Spinning & $\begin{array}{l}\text { CytoMate and } \\
\text { LOVO }\end{array}$ & $\begin{array}{l}\text { Effectively removes DMSO (CytoMate }{ }^{72} \text { and } \\
\text { LOVO) }{ }^{75} \text { washes and recovers viable CIK } \\
\text { cells from culture (CytoMate); }{ }^{71} \text { can wash } \\
\text { mobilized peripheral blood leukapheresis } \\
\text { products for CD34+ cells (LOVO); }{ }^{75} \\
\text { recovers inactivated in vitro-derived } \\
\text { platelets from nucleated cells (LOVO) }{ }^{76}\end{array}$ & $\begin{array}{l}\text { No protocol in place for washing and } \\
\text { volume reducing whole blood products; } \\
\text { requires large capital investment upfront }\end{array}$ \\
\hline Novel & \multicolumn{2}{|c|}{ Sedimentation, inertial focusing } & $\begin{array}{l}\text { Effectively removes } 80 \% \text { of free hemoglobin } \\
\text { and } 90 \% \text { of total free protein from stored } \\
\text { RBC units; efficiently reduces IgA to levels } \\
\text { safe for IgA-deficient recipients; does not } \\
\text { lead to significant buildup of potassium and } \\
\text { free hemoglobin during } 24 \text {-hour post-wash } \\
\text { storage time; low cost and portable }{ }^{81-83}\end{array}$ & $\begin{array}{l}\text { Has only been tested using small } \\
\text { volumes }(\sim 50 \mathrm{~mL}) ; \text { further research and } \\
\text { development needed to compete with } \\
\text { current washing systems }\end{array}$ \\
\hline
\end{tabular}

Abbreviations: CATS, continuous autotransfusion system; CIK, cytokine-induced killer; DMSO, dimethyl sulfoxide; PAF, potassium absorbing filters; RBCs, red blood cells; WBCs, white blood cells.

RBC hemolysis increases steadily over the 24-hour post-wash storage, ${ }^{17,23}$ calling for a reduction in expiration time from 24 hours to 6 hours. For patients receiving these washed units, the excess free hemoglobin can exacerbate clinical complications and lead to more negative prognoses. For cryopreserved blood products (eg, hematopoietic stem cells [HSC]), dimethyl sulfoxide (DMSO) that is used to prevent the formation of ice crystals must be washed off prior to transfusion because of its toxicity. ${ }^{10}$ Although cryopreserved products are typically washed using automated cell processors like COBE 2991, the system is unable to sufficiently reduce the DMSO concentration in a single run. ${ }^{24}$

Unlike open cell-processing systems, closed systems, such as the ACP 215 (Haemonetics Corporation, Braintree, 
MA, USA), significantly reduce the risk of bacterial contamination and allow for the addition of storage media to washed units. The addition of storage media prolongs the shelf life of washed RBCs from 24 hours to 7 days, while still meeting all standard regulatory metrics for washed units. ${ }^{25,26}$ In clinical studies, the use of the ACP 215 extended the shelf life of RBC units and led to a decrease in the number of units that had to be discarded due to expiration. ${ }^{25}$ Unfortunately, both COBE 2991 and ACP 215 were found to have caused significant RBC damage, indicating that centrifugation-based methods of washing with cell processors can reduce the quality of washed RBCs. Irradiating RBCs is also known to cause similar membrane damage, potassium leakage, and hemolysis, ${ }^{27}$ even though the technique is necessary to prevent transfusion-associated graft-versus-host disease and is indicated for neonates. Imposing additional stress on irradiated blood products by centrifugation may also worsen the quality of irradiated products further. ${ }^{23,28,29}$

Centrifugation-based cell processors are unable to consistently reduce the concentration of $\operatorname{IgA}$, below the level acceptable for transfusion to IgA-deficient patients, in a single-wash run. While washing RBCs twice with the ACP 215 led to a significant decrease in $\operatorname{IgA}$, incorporating a second washing step introduced further damage to the cells and increased the total processing time. ${ }^{30}$ Additional evidence suggests that washed deglycerolized RBCs have hemolysis levels that are not consistently within the range of regulatory standards after washing. ${ }^{31}$ ACP 215 has also been used to wash and concentrate platelets with a reported $99 \%$ plasma removal rate, compared to manual centrifugation, which yielded 95\%-97\% plasma removal. ${ }^{32,33}$ However, the intense mechanical stress associated with passing through a centrifugation-based cell processor inevitably activates platelets $^{34}$ as well as residual WBCs. ${ }^{35}$ These activated cells adhere to parts of the centrifugation chamber and tubing, and continually release cytokines and pro-inflammatory markers ${ }^{36}$ into the suspending medium of the washed blood product. Neither COBE 2991 nor ACP 215 can remove these activated cells and inflammatory markers, consequently diminishing the quality of washed blood products and causing a range of adverse outcomes at the point of care. ${ }^{37}$

\section{Autotransfusion systems: discontinuous and continuous flow}

Intraoperative blood salvage for autotransfusion decreases the need for allogeneic blood through the recovery and subsequent reinfusion of a patient's own blood shed during surgery. Blood salvage is particularly useful when a substantial
$(>20 \%)$ blood loss is anticipated, such as in cardiothoracic or orthopedic surgery, and during transplantations. ${ }^{38,39}$ Autotransfusion is also utilized for patients who cannot accept donated blood for cultural or religious reasons. ${ }^{39}$ During the cell salvage process, shed blood is aspirated from the surgical site and collected in a container with anticoagulant (heparin or citrate). Because the blood is suctioned from the surgical cavity and blood loss varies greatly depending on the patient and the type of surgical procedure, a wide range of contaminants (eg, fat, coagulation factors, skin and bone cells, excess plasma, and colloid solution used for fluid resuscitation) are also collected with shed blood. Therefore, salvaged blood is filtered, and then washed and volume reduced to generate a unit of concentrated RBCs before it can be reinfused back into the patient. Because of this functionality, the machines used for processing salvaged RBCs can also be used for washing and volume reduction of allogeneic cellular blood products.

A typical autotransfusion device suctions blood into a bowl (eg, Latham, Baylor, or disk shaped) which accommodates the variable intake of blood during the salvage process. Because the blood is exposed to air and high pressure during suction, massive RBC hemolysis and activation of platelets and WBCs may occur. Activated cells adhere to the surface of the bowl during centrifugation and release leukoattractants and thromboplastic materials into the unit. ${ }^{37}$ In a comparative study, whole blood washed with a discontinuous-flow autotransfusion device, Cell Saver Elite (Haemonetics Corporation), was found to have more hemolysis and microparticle formation than blood washed with COBE 2991, ${ }^{40}$ likely from the exposure of blood to both air and high suction pressures. However, in terms of potassium, free hemoglobin, platelet, and WBC removal, autotransfusion machines, such as the Cell Saver 5 (Haemonetics Corporation), have demonstrated a level of performance on par with conventional cell processors. ${ }^{37}$

The washing/volume reduction performance varies among autotransfusion devices even when they used the same Latham-bowl technology - for example, the Cell Saver 5 produces RBCs with lower hematocrits while achieving a higher RBC recovery than Sequestra 1000 (Medtronic, Minneapolis, MN, USA). ${ }^{41}$ Still, an even more significant source of variability in cell recovery rates is the ability to manually set and change centrifugation parameters, ${ }^{42}$ and therefore, the ultimate impact from processing on the quality of washed $\mathrm{RBC}$ product would likely depend on user preferences.

Autotransfusion devices based on the pneumatic disk technology, such as the OrthoPAT (Haemonetics Corporation), have a disposable disk of tunable size that allows for 
optimal processing of salvaged blood regardless of volume. Although these devices can process smaller volumes, the washing procedure typically takes longer than when performed using the conventional Latham bowl-based devices. ${ }^{43,44}$ Additionally, both pneumatic disk and Latham bowl-based discontinuous-flow devices retain a significant volume of blood in the bowl and tubing, ${ }^{43,44}$ which further decreases the yield of washed RBCs. Regardless, the ability to process small volumes of blood significantly expands the applicability of autotransfusion devices. ${ }^{43}$

Unlike the discontinuous-flow devices that must employ multiple disposable inserts to wash any volume of blood larger than the bowl size, continuous-flow technologies can fill, wash, and separate blood components continuously in a single disposable washing chamber. Fresenius Continuous Autotransfusion System (CATS; Terumo BCT) is the first Food and Drug Administration approved and commercially available autotransfusion device that washes and separates salvaged RBCs via continuous-flow centrifugation. Blood is driven through the machine and allowed to separate in a rotating double spiral washing chamber. During rotation, $\mathrm{RBCs}$ are forced into the outer wall of the spiral, while all other components move towards the inner wall. Platelets, plasma, and WBCs are removed as washing solution (eg, normal saline) is added to the concentrated RBCs. CATS can concentrate RBCs from whole blood or from stored RBC units before resuspension in normal saline to the desired hematocrit (and can also separate platelet-rich plasma from whole blood and concentrate platelets). The CATS disposable washing chamber requires a minimum fill volume of 30 $\mathrm{mL}$, allowing small-volume washing without any significant modifications to the system. Since the chamber is constantly rotating during processing, there are virtually no residual RBCs left in the washing chamber, providing a better recovery than conventional cell processors and autotransfusion devices that utilize a bowl or pneumatic disk.

In a direct comparison, CATS system generated washed RBCs with unchanged potassium levels and little hemolysis, unlike units washed with the COBE $2991 .{ }^{45}$ CATS was also able to produce $\mathrm{RBC}$ concentrates at a higher hematocrit than COBE 2991 and most other conventional discontinuous-flow autotransfusion devices. ${ }^{37,45}$ When testing for the removal of fat cells from salvaged blood, CATS surpassed discontinuous systems (eg, Cell Saver 5) and was on par with the Cell Save Elite featuring an integrated fat reduction program. ${ }^{46}$ Although the CATS system represents a significant improvement upon current centrifugation-based cell processors and discontinuous-flow autotransfusion devices, it is not without limitations. Washing RBCs with CATS yields inconsistent levels of free hemoglobin removal ${ }^{41}$ and has a negative effect on both RBC deformability and cell 2,3-DPG content after processing. ${ }^{47}$ Similar to other centrifugation-based cell processors, the negative effects of CATS processing on $\mathrm{RBC}$ properties may increase in a time-dependent manner following the washing procedure. ${ }^{48}$

Although autotransfusion devices are traditionally used to wash fresh blood shed during surgery, washing stored RBCs via autotransfusion has also been explored. Washing with the discontinuous-flow Dideco Electa 740E (Dideco SPA, Mirandola, Italy) aged stored RBCs. De Vroege et al found that ATP and 2,3-DPG contents of RBCs were reduced after washing. Furthermore, while there was free hemoglobin in the waste bag, free hemoglobin levels were not reduced in the final washed unit. ${ }^{49}$ This indicated that the washing procedure potentially damaged and lysed some RBCs resulting in the release of intracellular hemoglobin. It is possible that the constant deterioration RBCs undergo in storage makes them more susceptible to lysis during centrifugation-based processing. Interestingly, washing stored RBCs with the Cell Saver also did not reduce the amount of microparticles in the final unit, ${ }^{50}$ while CATS system was able to remove microaggregates with a wide range of sizes. ${ }^{50}$ Considering autotransfusion technologies were developed to wash relatively fresh salvaged blood, their design and default settings may not be optimal for washing stored RBCs. Furthermore, autotransfusion utilizes specific spinning regimes to remove contaminants not typically found in stored RBC units (eg, fat, bone, etc) and, therefore, may not be completely safe for stored, fragile RBCs.

Finally, autotransfusion devices have also been employed to separate platelets from platelet-rich plasma. Three types of autotransfusion devices utilizing either a Latham bowl, Baylor bowl, or closed-spiral chamber, showed only $50 \%-60 \%$ platelet recovery, ${ }^{51}$ which was consistently lower than that for conventional centrifugation. ${ }^{52}$ The platelet recovery performance of autotransfusion devices will have to be significantly improved before they could provide a practical alternative to specialized cell processors or manual centrifugation.

\section{Centrifugation-free technologies Membrane filtration}

Membrane filtration technologies present an attractive alternative to the centrifugation-based cell processors for volume reduction and washing of blood products because membranebased filtration systems typically do not have moving parts and are significantly simpler and less expensive. Simple 
filtration, such as using a hollow fiber membrane cartridge, is usually employed for filtering out excessive amounts of the suspending media (such as washing solution or plasma) and retaining the blood cells in suspension. A hollow fiber device, the PSN 120 (Baxter International Inc., Deerfield, IL, US) made from polysynthane, was successfully used to remove $95 \%$ of DMSO from cryopreserved platelets, showing a level of performance similar to that of the conventional centrifugation-based washing. ${ }^{53}$ In comparison, a cellulose-acetate dialyzer (\#23.05; Baxter International Inc.) was only able to remove $52 \%$ of DMSO from cryopreserved platelets, highlighting the importance of the material that the hollow fiber device employs. ${ }^{53}$ Both polysynthane and polyacrylonitrile-based ( $800 \mathrm{HF}$, AKZO; Mission Medical, Fremont, CA, USA) hollow fibers were found to cause a level of platelet activation (assessed via P-selectin expression) similar to that of conventional centrifugation. ${ }^{53}$ It is likely, therefore, that hollow fiber filtration devices made of these materials may also be suitable for washing and volume reducing conventional platelet concentrates.

Brune et al presented a gravity-based, hollow fiber filtration system utilizing a Sangofer (Sangofer neonatal; Heim Group, Gladbeck, Germany) hollow fiber system for separating RBCs from placental whole blood of full-term newborns. Approximately $60 \mathrm{~mL}$ or more of blood from a donation bag flows, via gravity, through a leukoreduction filter and then passes through a hollow fiber system that separates plasma from RBCs. The $\mathrm{RBCs}$ reached a final hematocrit of $\sim 56 \%$ and were on par, in terms of quality, with adult RBCs, both stored for 35 days. ${ }^{54}$ Based on this early work, ErySep Classic Blood Separation System (Lmb Technologie GmbH, Schwaig, Germany) and 3M Blood Separation System (3M, Maplewood, MN, USA) are two commercially available hollow fiber systems for separating RBCs and plasma from whole blood. Both the ErySep and 3M Blood Separation Systems operate by gravity and consist of a sampling bag, a whole blood bag, and two collection bags for RBCs and plasma, as well as an attached leukoreduction filter. $3 \mathrm{M}$ can process a unit of whole blood within 64 minutes and produce a $293-\mathrm{mL}$ RBC unit. ${ }^{55}$ ErySep takes $\sim 140$ minutes to produce a 306-mL RBC unit. ${ }^{56}$ Although RBCs met all regulatory standards, platelets are completely lost during processing, and plasma produced using these hollow fiber systems has significantly diminished concentrations of important clotting factors (eg, von Willebrand factor, antithrombin III, factor V, factor VIII, and factor XI). ${ }^{55-58}$ This important limitation notwithstanding, it is likely that these hollow fiber systems based on polyethersulfone membranes could be readily adapted for washing or volume reducing stored RBCs.
Recent developments in microfiltration utilize membranes for the absorption of unwanted solutes located in solution, such as excess potassium, free hemoglobin, and clotting residuals. Potassium absorbing filters (PAF), developed by Yamada et al have been shown to effectively reduce the amount of potassium in packed RBCs, decreasing the risk of hyperkalemia posttransfusion. ${ }^{59}$ Such a filter could ameliorate the buildup of potassium that occurs after centrifugation or irradiation. ${ }^{60}$ These filters have also been shown to remove excess ammonium from RBC units (76\%-87\% reduction), fresh frozen plasma $(21 \%-31 \%$ reduction $)$, platelets $(53 \%$ reduction), and albumin solutions (49\%-92\% reduction) reducing the risk of ammonium acting as a neurotoxin during traumatic brain injury or in neonates with underdeveloped blood-brain barriers. ${ }^{61}$ However, PAF filters are not currently compatible with rapid blood transfusion devices, and more research is still needed to determine the effect of PAF filtration on RBC quality. ${ }^{62,63}$ Although evidence is limited on the clinical feasibility and advantages of incorporating PAF filtration to standard blood transfusions, the apparent permissible flow rates could allow for high-throughput blood banking applications beyond the specific clinical significance of this technology for neonatal and pediatric hematology practices.

HemoSep (Brightwake, Nottinghamshire, UK) is a hemoconcentration system developed to soak up to $250 \mathrm{~mL}$ of excess plasma during blood salvage without the use of centrifugation. ${ }^{64-66}$ The primary component of the HemoSep system is a blood bag separated into two compartments by a filter membrane, which prevents cells in the blood compartment of the bag from entering the super-absorber pad contained in the other compartment of the bag. The blood plasma and other fluids are free to pass through the membrane, however, to be soaked up by the super-absorber pad. Robertson et al developed a sensor capable of measuring the hematocrit of packed RBCs, further increasing the utility of HemoSep hemoconcentration system. ${ }^{67}$ The low-cost, simplicity, and portability of the HemoSep system in comparison to conventional autotransfusion devices makes it an attractive alternative for blood salvage, particularly in resource-limited and military settings. ${ }^{6}$ Furthermore, HemoSep has been shown to provide a superior platelet recovery in a direct comparison to CATS (although CATS system was found to concentrate RBCs significantly better than HemoSep). ${ }^{69}$ Systems implementing physical filters for washing or volume reduction of RBCs (such as the HemoSep bag) provide an exciting alternative to conventional centrifugation-based machines, while also potentially reducing the damage to cells during processing. 


\section{Spinning membrane filtration}

Filtration using spinning membrane is another method used for blood cell fractionation. Spinning membrane separators provide excellent filtration rates by generating Taylor vortices in the gap between the membrane and the shell of the device. This unique flow pattern creates flow at the membrane, constantly sweeping the surface to prevent the cellular components from depositing on and clogging or fouling the membrane, while continually replenishing the medium to be filtered. ${ }^{70}$ CytoMate (Baxter International Inc.) is an automatic cell washing device that utilizes a spinning membrane to ensure cell filtration against a counter-flow buffer circulation..$^{71}$ The closed system has automatic userdefinable programming that allows for efficient separation with low risk of contamination. ${ }^{72}$ CytoMate has been used for a wide variety of applications such as for washing and recovery of viable cytokine-induced killer cells after extended culture, ${ }^{71}$ for removal of cryoprotective agents from HSC before infusion, ${ }^{72}$ and for reducing platelet contamination prior to selection of immunomagnetic $\mathrm{CD} 34+$ stem cells used in autologous transplants. ${ }^{73}$ CytoMate was shown to reproducibly remove $96 \%$ or more of DMSO from frozen $\mathrm{HSC}^{74}$ With such a high efficiency, it is conceivable that CytoMate could also be very effective for washing and volume reducing cellular blood components as well, although a special protocol will likely need to be developed to limit RBC lysis and platelet activation within the system.

The LOVO (Fresenius Kabi, Bad Homburg vor der Höhe, Germany) device similarly incorporates a non-pelletizing spinning membrane, made of $4-\mu \mathrm{m}$ track-etched polycarbonate, to rapidly separate out target cells, remove supernatant, and perform dilution or concentration of cells. ${ }^{75}$ LOVO has been utilized to achieve automated washing and purification of various cellular products. Schlinker et al adapted the LOVO device to separate in vitro-derived platelets from nucleated cells and later recover immature megakaryocytes for expansion in culture. ${ }^{76}$ Following membrane filtration, they were able to obtain platelets with high purity and low activation, and good recovery of megakaryocytes. LOVO has also been used to wash mobilized peripheral blood leukapheresis products for $\mathrm{CD} 34+$ cell isolation ${ }^{77}$ and to remove the cryoprotectant from hematopoietic progenitor cell grafts for bedside infusion. ${ }^{75}$ The use of LOVO for volume reduction and washing of classical transfusion products (RBCs, platelets, and granulocytes) may significantly reduce the cellular damage associated with centrifugation-based processing. However, similar to the centrifugation-based cell processors, LOVO requires a relatively large capital investment and costly disposable cartridges to operate, and therefore, may not be available in resource-limited settings.

\section{Novel centrifugation-free technologies}

Washing stored RBCs through centrifugation-free methods can prove challenging since RBCs have a dynamically changing shape and can pass through very narrow pores. ${ }^{78}$ This makes size-based filtration for washing and volume reduction of RBCs difficult to achieve. Many methods to filter out RBCs without centrifugation use an aggregation agent, like hydroxyethyl starch or dextran..$^{79,80}$ These agents accelerate aggregation of RBCs, allowing them to form rouleaux and sediment faster in normal gravity at the bottom of the container, while leaving platelet-rich plasma at the top layer. Our laboratory has developed a washing system that utilizes RBC sedimentation in normal gravity (but does not require any added aggregation agents) to wash stored RBCs, producing a product with a final hematocrit of about $37 \%$ while also removing $80 \%$ of free hemoglobin and $90 \%$ of total free protein. ${ }^{81}$ In this system, a dilute suspension of stored RBCs is flowing through a coiled narrow bore tubing, oriented in such a way as to allow RBC sedimentation perpendicular to the direction of flow. A series of bifurcations built-in along the length of the tubing siphons off washing waste while allowing sedimented RBCs to continue flowing in the tubing until they are collected at the end of the system.

Further development has made the next generation of our centrifugation-free washing system significantly faster and more efficient, by incorporating "inertial focusing" (to enable high-throughput separation of washing solution from washed RBCs), and by adding a membrane pouch with a biocompatible hydrogel to absorb the excess fluid and concentrate washed RBCs to the desired hematocrit. ${ }^{82}$ This washing system was able to reduce IgA level to about $0.02 \mathrm{mg} / \mathrm{dL}$ in a single pass, ${ }^{82}$ while conventional centrifugation-based washing typically requires two cycles to reduce IgA levels to a concentration that is safe for IgA deficient recipients $(<0.05 \mathrm{mg} / \mathrm{dL}) .{ }^{30}$ Importantly, our centrifugation-free washing system significantly reduced the buildup of extracellular potassium and free hemoglobin in the supernatant of washed RBCs during the 24-hours short-term storage. In fact, the levels of potassium and free hemoglobin in samples washed using our system after 24 hours were as good as in paired samples immediately after centrifugation-based washing. ${ }^{83}$ Excess potassium and free hemoglobin are markers of RBC damage and deterioration, and our findings, therefore, indicate that washing stored RBCs with our centrifugation-free system leads to less damaged RBCs in comparison to conventional centrifugation-based cell processing. 
While more research and development is needed before our centrifugation-free washing system becomes truly competitive, it may address key limitations of centrifugationbased washing systems, including high cost, complexity, and the significant damage to cells occurring during centrifugation. Our system also has several important advantages over existing centrifugation-free approaches. For example, unlike hollow fiber filtration, our system is able to remove, concurrently, both large contaminants (eg, microparticles and cellular debris) and soluble contaminants (eg, potassium, free hemoglobin, and IgA) when washing stored RBCs. An important limitation of our work so far is that our centrifugationfree washing system has been tested only with relatively small volumes of blood $(\sim 50 \mathrm{~mL})$, and increasing the capacity (and throughput) of the system further will require multiplexing several of the separation tubing coils (which is a nontrivial engineering task). However, even the current small-volume prototype of our system could be useful for neonatal and infant patients, who require relatively small volumes when transfused. For these patients, conventional washing using most centrifugation-based cell processors is not practical due to the large dead volume within the disposable sets.

\section{Conclusion}

The washing and volume-reduction of blood products may prevent a host of clinical complications caused by the adverse effects associated with residual plasma proteins, and other contaminants found in units of blood component after processing and storage. Here, we reviewed several traditional cell processing techniques based on centrifugation, including manual centrifugation, automated cell processors, and various autotransfusion devices. Although these methods are commonly used to process blood, there is accumulating evidence that centrifugation leads to increased hemolysis, activation of platelets and leukocytes, cellular damage, and various other negative effects that reduce the quality and efficacy of cellular blood products. We also discussed several classes of centrifugation-free approaches, including membraneand inertia-based separation systems that aim to produce high-quality washed or volume reduced blood products with minimal cellular damage. While these centrifugation-free approaches are very promising, they typically serve niche applications and many are still in early stages of development. Further research is needed to move these emerging technologies into the mainstream of blood cell processing, to provide the highest quality blood products for transfusion and ultimate improve clinical outcomes for the patients.

\section{Acknowledgments}

This work was supported in part by a 2012 NIH Director's Transformative Research Award (NHLBI R01HL117329, PI: SSS). Study sponsor had no role in the writing of the article, and in the decision to submit the paper for publication.

\section{Disclosure}

The authors report no conflicts of interest in this work.

\section{References}

1. Cholette JM, Pietropaoli AP, Henrichs KF, et al. Longer RBC storage duration is associated with increased postoperative infections in pediatric cardiac surgery. Pediatr Crit Care Med. 2015;16(3):227-235.

2. Remy KE, Hall MW, Cholette J, et al. Mechanisms of red blood cell transfusion-related immunomodulation. Transfusion. 2018;58(3):804-815.

3. Karafin MS, Carpenter E, Pan A, Simpson P, Field JJ. Older red cell units are associated with an increased incidence of infection in chronically transfused adults with sickle cell disease. Transfusion and Apheresis Science. 2017;56(3):345-351.

4. Kent MW, Kelher MR, West FB, Silliman CC. The pro-inflammatory potential of microparticles in red blood cell units. Transfus Med. 2014;24(3):176-181.

5. Makroo RN, Raina V, Bhatia A, et al. Evaluation of the red cell hemolysis in packed red cells during processing and storage. Asian JTransfus Sci. 2011;5(1):15.

6. Flatt JF, Bawazir WM, Bruce LJ. The involvement of cation leaks in the storage lesion of red blood cells. Front Physiol. 2014;5(365):214.

7. Crighton GL, Estcourt LJ, Wood EM, et al. A therapeutic-only versus prophylactic platelet transfusion strategy for preventing bleeding in patients with haematological disorders after myelosuppressive chemotherapy or stem cell transplantation. Cochrane Database Syst Rev. 2015;12(10):CD010981.

8. Anani W, Triulzi D, Yazer MH, Qu L. Relative IgA-deficient recipients have an increased risk of severe allergic transfusion reactions. Vox Sang. 2014;107(4):389-392.

9. Tobian AA, Savage WJ, Tisch DJ, Thoman S, King KE, Ness PM. Prevention of allergic transfusion reactions to platelets and red blood cells through plasma reduction. Transfusion. 2011;51(8):1676-1683.

10. Chen C, Sharma S, Doo H. Washing stem cells cryopreserved in Dimethyl Sulfoxide (DMSO) before infusion prevents DMSO-related side effects with little loss of CD34 positive cells and prompt engraftment. Am Soc Hematology. 2004.

11. Abonnenc M, Pesse B, Tissot JD, Barelli S, Lion N. Automatic washing of thawed haematopoietic progenitor cell grafts: a preclinical evaluation. Vox Sang. 2017;112(4):367-378.

12. Hirayama J, Azuma H, Fujihara M, et al. Comparison between bacterial growth in platelets (PLTs) washed with M-sol and that in PLT-rich plasma. Transfusion. 2011;51(7):1592-1594.

13. Schoenfeld H, Muhm M, Doepfmer UR, Kox WJ, Spies C, Radtke $\mathrm{H}$. The functional integrity of platelets in volume-reduced platelet concentrates. Anesth Analg. 2005;100(1):78-81.

14. Veeraputhiran M, Ware J, Dent JA. A comparison of washed and volumereduced platelets with respect to platelet activation, aggregation, and plasma protein removal. Transfusion. 2010;50.

15. Fernández-Muñoz H, Castilla-Llorente C, Plaza EM, et al. Quality assessment and transfusion efficacy of buffy coat-derived platelet concentrates washed with platelet additive solution. Blood Transfus. 2018;16(3):273.

16. Brambilla M, Rossetti L, Zara C, et al. Do methodological differences account for the current controversy on tissue factor expression in platelets? Platelets. 2018;29(4):406-414. 
17. O’Leary MF, Szklarski P, Klein TM, Young PP. Hemolysis of red blood cells after cell washing with different automated technologies: clinical implications in a neonatal cardiac surgery population. Transfusion. 2011;51(5):955-960.

18. Rapido F, Brittenham GM, Bandyopadhyay S, et al. Prolonged red cell storage before transfusion increases extravascular hemolysis. J Clin Invest. 2017;127(1):375-382.

19. Swindell CG, Barker TA, Mcguirk SP, et al. Washing of irradiated red blood cells prevents hyperkalaemia during cardiopulmonary bypass in neonates and infants undergoing surgery for complex congenital heart disease. Eur J Cardiothorac Surg. 2007;31(4):659-664.

20. Lee AC, Reduque LL, Luban NL, Ness PM, Anton B, Heitmiller ES. Transfusion-associated hyperkalemic cardiac arrest in pediatric patients receiving massive transfusion. Transfusion. 2014;54(1):244-254.

21. Cholette JM, Henrichs KF, Alfieris GM, et al. Washing red blood cells and platelets transfused in cardiac surgery reduces post-operative inflammation and number of transfusions: Results of a prospective, randomized, controlled clinical trial. Pediatr crit care med. 2012;13(3):290-299.

22. Cortés-Puch I, Wang D, Sun J, et al. Washing older blood units before transfusion reduces plasma iron and improves outcomes in experimental canine pneumonia. Blood. 2014;123(9):1403-1411.

23. Weiskopf RB, Schnapp S, Rouine-Rapp K, Bostrom A, Toy P. Extracellular potassium concentrations in red blood cell suspensions after irradiation and washing (vol 45, pg 1295, 2005). Transfusion. 2006;46:1461.

24. Chiche-Lapierre CE, Tramalloni D, Chaput N, Lapierre V. Comparative analysis of sepax S-100, COBE 2991, and manual DMSO removal techniques from cryopreserved hematopoietic stem cell apheresis product. Cytotherapy. 2016;18(6):S47.

25. Hansen A, Yi Q-L, Acker JP. Quality of red blood cells washed using the ACP 215 cell processor: assessment of optimal pre- and postwash storage times and conditions. Transfusion. 2013;53(8):1772-1779.

26. Acker JP, Hansen AL, Yi QL, et al. Introduction of a closed-system cell processor for red blood cell washing: postimplementation monitoring of safety and efficacy. Transfusion. 2016;56(1):49-57.

27. Harm SK, Raval JS, Cramer J, Waters JH, Yazer MH. Haemolysis and sublethal injury of RBCs after routine blood bank manipulations. Transfus Med. 2012;22(3):181-185.

28. Weiss DR, Goehring J, Weisbach V, et al. Centrifugation after irradiation of red blood cells does not accelerate haemolysis. Clin Lab. 2011;57(7-8):523-526.

29. Serrano K, Pambrun C, Levin E, Devine DV. Supernatant reduction of stored gamma-irradiated red blood cells minimizes potentially harmful substances present in transfusion aliquots for neonates. Transfusion. 2017;57(12):3009-3018.

30. Hansen AL, Turner TR, Kurach JD, Acker JP. Quality of red blood cells washed using a second wash sequence on an automated cell processor. Transfusion. 2015;55(10):2415-2421.

31. Turner T, Hansen A, Kurach J, Acker JP. From development to implementation: adjusting the hematocrit of deglycerolized red cell concentrates to meet regulatory standards. Transfusion Medicine and Hemotherapy. 2017;44(1):30-38.

32. Iwama A, Hirayama J, Nogawa M, et al. Comparison between in vitro properties of washed platelet concentrates suspended in M-sol and those in BRS-A, both of which were prepared with an automated cell processor. Transfusion and Apheresis Science. 2017;56(2):241-244.

33. Oikawa S, Minegishi M, Endo K, Kawashima W, Suzuki K, Shimizu H. Washing of platelets can be fully automated using a closedsystem cell processor and BRS-A platelet additive solution. Vox Sang. 2016;111(4):437-440.

34. Söderström AC, Nybo M, Nielsen C, Vinholt PJ. The effect of centrifugation speed and time on pre-analytical platelet activation. Clinical Chemistry and Laboratory Medicine. 2016;54(12):1913-1920.

35. Dohan Ehrenfest DM, Pinto NR, Pereda A, et al. The impact of the centrifuge characteristics and centrifugation protocols on the cells, growth factors, and fibrin architecture of a leukocyte- and platelet-rich fibrin (L-PRF) clot and membrane. Platelets. 2018;29(2):171-184.
36. Schneider SO, Rensing H, Hartmann L, Grundmann U, Volk T, Biedler A. Impact of intraoperatively salvaged and washed blood on stimulated cytokine release in vitro. Transfusion. 2014;54(10pt2):2782-2790.

37. Serrick CJ, Scholz M. Partial bowls using the Haemonetics Cell Saver 5: does it produce a quality product? J Extra Corpor Technol. 2005;37(2):161.

38. Yoon C, Noh S, Lee JC, Ko SH, Ahn W, Kim HC. Influence of the washing program on the blood processing performance of a continuous autotransfusion device. Journal of Artificial Organs. 2014;17(1):118-122.

39. Esper SA, Waters JH. Intra-operative cell salvage: a fresh look at the indications and contraindications. Blood Transfus. 2011;9(2):139.

40. Bennett-Guerrero E, Kirby BS, Zhu H, Herman AE, Bandarenko N, Mcmahon TJ. Randomized study of washing 40- to 42-day-stored red blood cells. Transfusion. 2014;54(10):2544-2552.

41. Serrick CJ, Scholz M, Melo A, Singh O, Noel D. Quality of red blood cells using autotransfusion devices: a comparative analysis. Journal of Extracorporeal Technology. 2003;35:28-34.

42. Naumenko KS, Kim SF, Cherkanova MS, Naumenko SE. The Haemonetics Cell Saver 5 washing properties: effect of different washing pump and centrifuge speeds. Interact Cardiovasc Thorac Surg. 2008;7(5): 759-763.

43. Waters JH, Tuohy MJ, Hobson DF, Procop G. Bacterial reduction by cell salvage washing and leukocyte depletion filtration. Anesthesiology. 2003;99(3):652-655.

44. Seyfried T, Breu A, Gruber M, Reipert J, Hansen E. Processing of small volumes in blood salvage devices. Transfusion. 2014;54(10pt2): 2775-2781.

45. Sasaki J, Tirotta C, Lim H, et al. Comparison of stored red blood cell washing techniques for priming extracorporeal circuits. Perfusion. 2018;33(2):130-135.

46. Seyfried TF, Haas L, Gruber M, Breu A, Loibl M, Hansen E. Fat removal during cell salvage: a comparison of four different cell salvage devices. Transfusion. 2015;55(7):1637-1643.

47. Gu YJ, Vermeijden WJ, de Vries AJ, Hagenaars JA, Graaff R, van Oeveren W. Influence of mechanical cell salvage on red blood cell aggregation, deformability, and 2,3-diphosphoglycerate in patients undergoing cardiac surgery with cardiopulmonary bypass. Ann Thorac Surg. 2008;86(5):1570-1575.

48. Che J, Tian M, Ding G, et al. Effects of cell salvage on erythrocyte 2,3-disphosphoglycerate and G-6-PD levels and phosphatidylserine expression. Int J Lab Hematol. 2013;35(4):385-392.

49. de Vroege R, Wildevuur WR, Muradin JA, Graves D, van Oeveren W. Washing of stored red blood cells by an autotransfusion device before transfusion. Vox Sang. 2007;92(2):130-135.

50. Westphal-Varghese B, Erren M, Westphal M, et al. Processing of stored packed red blood cells using autotransfusion devices decreases potassium and microaggregates: a prospective, randomized, single-blinded in vitro study. Transfus Med. 2007;17(2):89-95.

51. Scholz M, Serrick C, Noel D, Singh O, Melo A. A prospective comparison of the platelet sequestration ability of three autotransfusion devices. J Extra Corpor Technol. 2005;37(3):286.

52. Seyfried TF, Streithoff F, Gruber M, et al. Platelet sequestration with a new-generation autotransfusion device. Transfusion. 2018;58(4): 989-997.

53. Arnaud F, Kapnik E, Meryman HT. Use of hollow fiber membrane filtration for the removal of DMSO from platelet concentrates. Platelets. 2003;14(3):131-137.

54. Brune T, Fill S, Heim G, Rabsilber A, Wohlfarth K, Garritsen HSP. Quality and stability of red cells derived from gravity-separated placental blood with a hollow-fiber system. Transfusion. 2007;47(12):2271-2275.

55. Hackstein H, Möller A, Gerlach M, Sachs U, Bein G. Prospective quality control study of a novel gravity-driven whole blood separation system suitable for humanitarian crises. Vox Sang. 2017;112(8):806-809.

56. Johnson L, Kwok M, Marks DC. Preparation of red blood cell concentrates and plasma units from whole blood held overnight using a hollowfibre separation system. Transfusion Medicine. 2015;25(1):13-19. 
57. Brune T, Hannemann-Pohl K, Nißle K, Ecker N, Garritsen H. Quality, stability, and safety data of packed red cells and plasma processed by gravity separation using a new fully integrated hollow-fibre filter device. Advances in Hematology. 2009;2009(3):1-6.

58. Hornsey VS, Mccoll K, Drummond O, Prowse CV. Separation of whole blood into plasma and red cells by using a hollow-fibre filtration system. Vox Sanguinis. 2005;89(2):81-85.

59. Yamada C, Heitmiller ES, Ness PM, King KE. Reduction in potassium concentration of stored red blood cell units using a resin filter. Transfusion. 2010;50(9):1926-1933.

60. Morrison A, Mcmillan L, Campbell JDM, Petrik J. Evaluation of a potassium removal filter on irradiated red cells stored in SAGM. Transfusion Medicine. 2015;25(5):320-325.

61. Fujita H, Shiotani Y, Takada Y, Nishimura S. Effects of potassium adsorption filters on the removal of ammonia from blood products. Blood Transfus. 2018;16(2):173.

62. Matsuura H, Akatsuka Y, Muramatsu C, et al. Evaluation of the potassium adsorption capacity of a potassium adsorption filter during rapid blood transfusion. Vox Sang. 2015;108(4):428-431.

63. Cid J, Villegas V, Carbassé G, Alba C, Perea D, Lozano M. Transfusion of irradiated red blood cell units with a potassium adsorption filter: A randomized controlled trial. Transfusion. 2016;56(5):1046-1051.

64. Gunaydin S, Gourlay T. Novel ultrafiltration technique for blood conservation in cardiac operations. The Annals of Thoracic Surgery. 2013;95(6):2148-2151.

65. Hogan M, Needham A, Ortmann E, et al. Haemoconcentration of residual cardiopulmonary bypass blood using Hemosep ${ }^{\circledR}$ : a randomised controlled trial. Anaesthesia. 2015;70(5):563-570.

66. Boyle G, Kuffel A, Parmar K, et al. A comparison of haemostatic biomarkers during low-risk patients undergoing cardiopulmonary bypass using either conventional centrifugal cell salvage or the HemoSep device. Perfusion. 2018;95:026765911878905.

67. Robertson CA, Gourlay T. Development of a diagnostic sensor for measuring blood cell concentrations during haemoconcentration. Perfusion. 2017;32(2):126-132.

68. Gourlay T, Simpson C, Robertson CA. Development of a portable blood salvage and autotransfusion technology to enhance survivability of personnel requiring major medical interventions in austere or military environments. J R Army Med Corps. 2018;164(2):96-102.

69. Gunaydin S, Robertson C, Budak AB, Gourlay T. Comparative evaluation of blood salvage techniques in patients undergoing cardiac surgery with cardiopulmonary bypass. Perfusion. 2018;33(2):105-109.

70. Wegener C, Olson B, Min K. Method for sized-based cell separation using spinning membrane filtration: Google Patents. 2017.
71. Giancola R, Olioso P, di Riti M, et al. Evaluation of an automated closed fluid management device for processing expanded cytokine-induced killer cells to use in immunotherapy programs for cancer. Transfusion. 2008;48(4):629-639.

72. Shu Z, Heimfeld S, Gao D. Hematopoietic SCT with cryopreserved grafts: adverse reactions after transplantation and cryoprotectant removal before infusion. Bone Marrow Transplantation. 2014;49(4):469-476.

73. Zinno F, Landi F, Aureli V, et al. Positive immunomagnetic CD34+ cell selection in haplo-identical transplants in $\beta$-thalassemia patients: removal of platelets using an automated system. Cytotherapy. 2010;12(1):60-66.

74. Calmels B, Houzé P, Hengesse JC, Ducrot T, Malenfant C, Chabannon C. Preclinical evaluation of an automated closed fluid management device: Cytomate, for washing out DMSO from hematopoietic stem cell grafts after thawing. Bone Marrow Transplant. 2003;31(9):823-828.

75. Mfarrej B, Bouchet G, Couquiaud J, et al. Pre-clinical assessment of the Lovo device for dimethyl sulfoxide removal and cell concentration in thawed hematopoietic progenitor cell grafts. Cytotherapy. 2017;19(12):1501-1508.

76. Schlinker AC, Radwanski K, Wegener C, Min K, Miller WM. Separation of in-vitro-derived megakaryocytes and platelets using spinning-membrane filtration. Biotechnology and Bioengineering. 2015;112(4):788-800.

77. Schlinker AC. Automated, spinning membrane filtration for preparation of mobilized leukapheresis products for CD34+ cell selection. Cell and Gene Therapy Insights. 2017;3(8):623-637.

78. Yang X, Forouzan O, Brown TP, Shevkoplyas SS. Integrated separation of blood plasma from whole blood for microfluidic paper-based analytical devices. Lab Chip. 2012;12(2):274-280.

79. Gannon T, Mcconnell K, Riley J. The use of hydroxy-ethyl starch sedimentation for autologous buffy coat preparation. J Extra Corpor Technol. 2005;37(3):311.

80. Muñoz M, García-Segovia S, Ariza D, Cobos A, García-Erce JA, Thomas D. Sedimentation method for preparation of postoperatively salvaged unwashed shed blood in orthopaedic surgery. Br J Anaesth. 2010;105(4):457-465.

81. Khanal G, Huynh RA, Torabian K, Xia H, Vörös E, Shevkoplyas SS. Towards bedside washing of stored red blood cells: a prototype of a simple apparatus based on microscale sedimentation in normal gravity. Vox Sang. 2018;113(1):31-39.

82. Vörös E, Piety NZ, Strachan BC, Lu M, Shevkoplyas SS. Centrifugationfree washing: A novel approach for removing immunoglobulin A from stored red blood cells. Am J Hematol. 2018;93(4):518-526.

83. Vörös E, Lu M, Lezzar D, Shevkoplyas SS. Centrifugation-free washing reduces buildup of potassium and free hemoglobin in washed red blood cells after the procedure. Am J Hematol. 2018;93(12):E389-E391.
Journal of Blood Medicine

\section{Publish your work in this journal}

The Journal of Blood Medicine is an international, peer-reviewed, open access, online journal publishing laboratory, experimental and clinical aspects of all aspect pertaining to blood based medicine including but not limited to: Transfusion Medicine; Blood collection, Donor issues, Transmittable diseases, and Blood banking logistics; Immunohematology; Artificial and alternative

\section{Dovepress}

blood based therapeutics; Hematology; Biotechnology/nanotechnology of blood related medicine; Legal aspects of blood medicine; Historical perspectives. The manuscript management system is completely online and includes a very quick and fair peer-review system. Visit http://www.dovepress.com/ testimonials.php to read real quotes from published authors. 